\title{
Cascade of Complications Following Carotid Body Tumor Excision
}

\author{
Pawan K Garg, MD ${ }^{1}$, Sarbesh Tiwari, MD, DM¹, Tushar S Ghosh, DNB, PDCC ${ }^{1}$, Surendra Patel, Mch², \\ Ankur Sharma, $\mathrm{MD}^{3}$, Pushpinder S Khera, $\mathrm{MD}^{1}$ \\ ${ }^{1}$ Department of Diagnostic and Interventional Radiology, All India Institute of Medical Sciences, Jodhpur, India \\ ${ }^{2}$ Department of Cardiothoracic Surgery, All India Institute of Medical Sciences, Jodhpur, India \\ ${ }^{3}$ Department of Anaesthesiology and Critical Care, All India Institute of Medical Sciences, Jodhpur, India
}

Carotid body tumor excision can lead to various complications including vascular injury and pseudoaneurysm formation. Here we describe a case of carotid body tumor excision followed by series of complications including pseudoaneurysm formation, failure of primary surgical repair, carotid stump syndrome following parent artery occlusion, and persistent hypotension.

Key Words: Carotid body tumor; Carotid artery injury; Carotid artery; Stroke

\author{
Correspondence to: \\ Sarbesh Tiwari, MD, DM \\ Department of Diagnostic and \\ Interventional Radiology, All India \\ Institute of Medical Sciences, Jodhpur, \\ Basni Industrial Area Phase-2, Jodhpur \\ 342005, Rajasthan, India \\ Tel: +918553990641 \\ Fax: +912912831961 \\ E-mail: sarbesh1984@gmail.com \\ Received: May 17, 2021 \\ Revised: August 10, 2021 \\ Accepted: September 8, 2021
}

Copyright $\odot 2021$ Korean Society of Interventional Neuroradiology

This is an Open Access article distributed under the terms of the Creative Commons Attribution Non-Commercial License (http://creativecommons.org/licenses/by-nc/4.0) which permits unrestricted non-commercial use, distribution, and reproduction in any medium, provided the original work is properly cited.

pISSN 2093-9043 eISSN 2233-6273 
neck swelling. Preoperative computed tomography (CT) with angiogram (Fig. 1A) revealed an intensely enhancing lesion, $4.3 \mathrm{~cm}$ in maximum dimension, abutting and splaying the internal carotid artery (ICA) and external carotid artery (ECA) with an angle of contact of more than 180 degrees, suggesting a Shamblin type II tumor. Local examination showed a large pulsatile swelling at the surgical site on the left side of the neck. His blood pressure was 110/74 (84) mm of $\mathrm{Hg}$ and his pulse rate was 96 per minute. Blood investigations including hemoglobin and white blood cell count were within normal limits.

A repeat $C T$ scan of the neck with angiography demonstrated a pseudoaneurysm at the stump of ligated ECA with surrounding hematoma (Fig. 1B). A diagnostic catheter angiogram showed narrowing of the distal common carotid artery (CCA) with small outpouching at ligated ECA origin with no active extravasation. He subsequently underwent neck exploration with excision of the pseudoaneurysm and primary repair of ECA rent.

During this period, he was diagnosed with type II diabetes mellitus. The neck wound developed an infection with Pseudomonas aeruginosa, which was managed conservatively. At the time of discharge on day 14 , he was having left marginal mandibular nerve palsy, deviation of the uvula to left, hoarseness of voice, and left-sided partial ptosis (Horner's syndrome), which possibly were surgical complications.

A week later on day 24 , he again presented with surgical wound site swelling. Repeat $\mathrm{CT}$ angiography of the neck revealed mild arterial wall irregularity at the previous operative site with adjoining hematoma. No definite pseudoaneurysm sac was found on the CT angiogram.

The next day (day 25), early morning active bleeding started from the wound site with hypotension; hence the plan was for catheter angiography. A diagnostic catheter angiogram showed contrast outpouching at the left CCA-ICA junction (suggestive of ruptured carotid pseudoaneurysm) with active extravasation (Fig. 1C). Since the patient had active bleeding with an infected wound and previously failed surgery, the decision for PAO was made. Balloon test occlusion (BTO) was done to look for cross flow and prognostication, though there was no other option to preserve the ICA. BTO showed good crossflow with a venous delay of less than 2 seconds with a small watershed perfusion defect in ACAMCA territory. Multiple coils were deployed into the left ICACCA across the pseudoaneurysm with PAO leaving behind a small left CCA stump (Fig. 1D). In the end, the check run showed no opacification or antegrade flow in the left ICA or ECA.

The patient was started on inotropes with the target to maintain the mean arterial pressure (MAP) in the range of
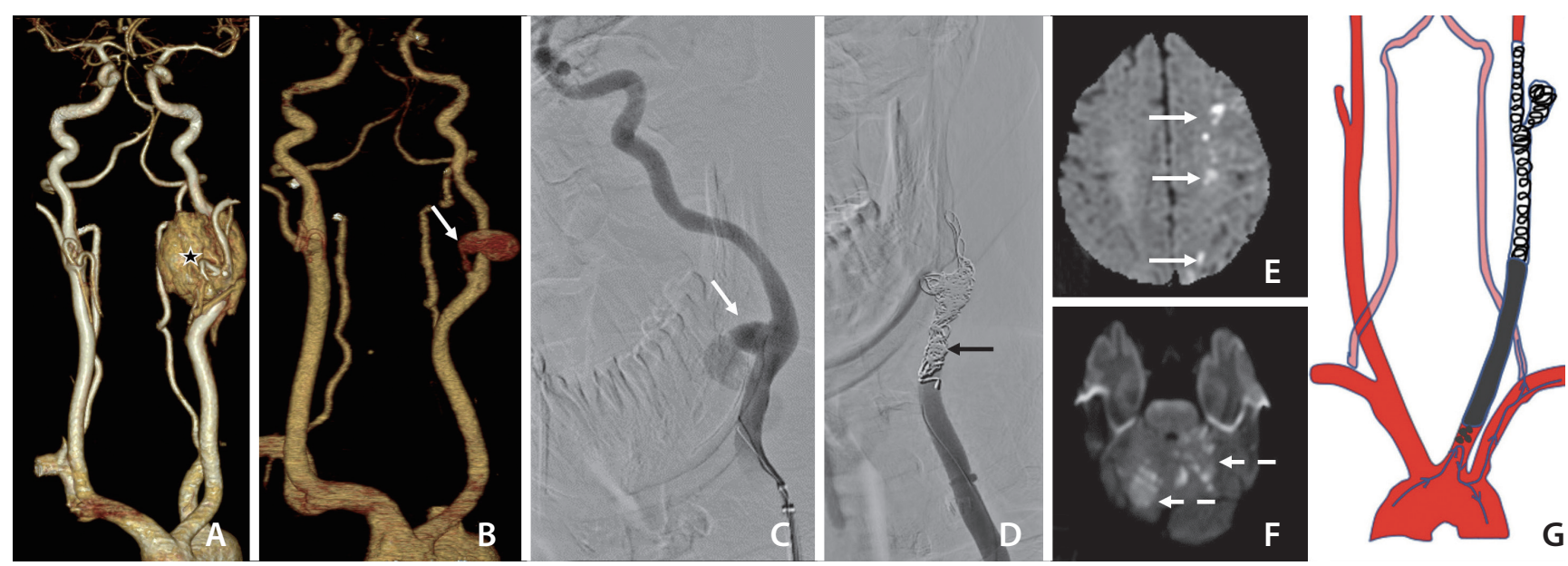

Fig. 1. (A) Computed tomography (CT) angiography baseline, volume rendering technique image showing left carotid body tumor (asterisk). (B) Day-8, post-operative CT showing a pseudoaneurysm (white arrow). (C) Day-25, left common carotid artery (CCA) angiogram confirmed the pseudoaneurysm arising from the medial wall with active contrast extravasation (white arrow). Balloon test occlusion (not shown) showed adequate cross flow across anterior communicating artery with the venous delay of less than 2 seconds. (D) Left CCA run showing occluded CCA by coils (black arrow). (E) Day-25, Post 6 hours of left CCA parent artery occlusion, magnetic resonance imaging (MRI) diffusion weighted image (DWI) at the level of corona radiata showing multiple foci of diffusion restriction (white arrows), suggestive of left-sided ACA-MCA territory watershed infarcts. (F) Day-26, MRI DWI at the level of pons showing multifocal patchy areas of diffusion restriction (dashed arrows), suggestive of acute infarcts involving posterior circulation. (G) Schematic diagram showing migration of emboli from left CCA stump to posterior circulation (left CCA stump $\rightarrow$ left subclavian artery $\rightarrow$ left vertebral artery $\rightarrow$ posterior circulation). 
100-110 mmHg. Nevertheless, a couple of hours later, he developed right-sided upper and lower limb weakness, and a magnetic resonance imaging (MRI) scan showed left ACAMCA watershed territory infarcts (Fig. 1E). At that time, the patient's MAP was in the range of 90-94 $\mathrm{mmHg}$, despite a high dose of single inotrope. Double inotropes were started along with high-volume saline infusion to further increase the MAP. The right-sided weakness improved significantly in the next few hours.

But during the next morning (day 26), he suddenly became drowsy and confused. At that time, he was on a highdose double inotrope with a MAP of 100-102 mmHg. An emergency MRI was done, which surprisingly revealed multiple new patchy foci of diffusion restriction involving the posterior circulation (bilateral cerebellar hemisphere, superior vermis, and occipital lobes) (Fig. 1F). No new area of infarction was seen in the anterior circulation in comparison to the previous day's MRI. He was managed with aspirin, anticoagulants (unfractionated heparin infusion), and maintenance of a high MAP.

Over time, his neurological symptoms improved completely and ionotropic support was tapered, but we could not wean him off completely as he was having prolonged and persistent hypotension. He also developed another episode of hemodynamic stroke while trying to take him off ionotropic support. We managed this prolonged hypotension with high volume intravenous fluid, inotropes, and postural management. Ionotropic support was gradually tapered over the next 4 weeks and he was discharged in a stable condition on the 55th day following the first neck surgery. All events are summarized in Supplementary Table 1.

\section{DISCUSSION}

CBT excision can be associated with complications like significant blood loss, vascular injury, pseudoaneurysm formation, and nerve injury. The complications depend upon the Shamblin type of CBT. A higher Shamblin type is associated with difficult surgery, more blood loss, and more chances of vascular injury. Preoperative embolization of a CBT reduces the blood loss and duration of surgery.'

The management of a pseudoaneurysm includes surgical repair or endovascular treatment. The surgical repair includes excision of a pseudoaneurysm with primary repair or interposition graft placement. The endovascular management includes placement of stent graft across the neck of a pseudoaneurysm or PAO after checking the crossflow. In case of an infected pseudoaneurysm or non-availability of the stent graft, the PAO is the only option in an emergency. Sylvester et al reported a neurological complication rate of $21.7 \%$ in a total 46 patients who underwent $\mathrm{PAO}$ of ICA. ${ }^{2}$

Rarely, after complete occlusion of the ICA, a short patent portion of the proximal ICA can be demonstrated by various imaging modalities and is referred to as the "carotid stump". 3-6 Classic CSS was first described by Field et al, who reported angiographic findings of collateral circulation between ECAICA circulation after ipsilateral ICA and PAO? Historically, classic CSS is described as recurrent episodes of ipsilateral cerebrovascular events in the form of acute ischemic embolic strokes due to migration of micro-emboli from the ipsilateral ICA stump to the ipsilateral patent ECA and then into intracranial circulation via patent ECA-ICA anastomosis. ${ }^{8-10}$

A case of CCA stump syndrome was described by Omoto et al. ${ }^{11}$, who reported a cerebral embolism in a case of chronically occluded CCA through the collateral flow from the superior thyroid artery to ICA. They demonstrated a mobile thrombus in the distal stump of the occluded CCA."

But even with an extensive literature search, we couldn't find any text mentioning CCA stump syndrome with ligated ECA. The possible mechanisms of these posterior circulation infarcts include emboli formed in the left CCA residual stump that eventually progressed to the posterior circulation via the left subclavian artery and left vertebral artery. (Fig. 1G).

Since the left ICA and ECA were completely occluded, the possibility of embolism via the posterior communicating artery and ECA to vertebral artery collaterals looked unlikely. ${ }^{12}$

It may be argued that in this process there must be symptoms of ischemia in the left upper extremity/digits, but the number of emboli needed to develop ischemic signs and symptoms in the left upper extremity will be much more than that for developing infarcts in the posterior fossa. And even if it did happen, it might not be evident because of rich collaterals in the hand and digits. As it was a single episode diagnosed immediately after the development of initial symptoms and managed with anticoagulants and antiplatelets, there were no further similar episodes.

Another event that happened in this case was prolonged persistent hypotension. We found a few reports documenting a decrease in blood pressure following unilateral CBT resection. ${ }^{13,14}$ All these studies, however, stated how unilateral CBT resection helps to improve blood pressure in 
patients with drug-resistant hypertension, but none of them mentioned its consequences in normotensive individuals. CBT is located at the carotid bifurcation and innervated by a parasympathetic (glossopharyngeal nerve) as well as sympathetic (superior cervical ganglion) nerve plexus. ${ }^{15}$ Surgical removal of non-functional CBT removes the stimulatory effect of chemoreflex on the sympathetic nervous system, but, at the same time, is also associated with opposing effects on sympathetic regulation by concomitant damage on the baroreceptors present in the carotid body. Unilateral CBT resection followed by neck exploration with pseudoaneurysm excision and left ICA-CCA PAO by coil mass could all have had a cumulative effect on the chemoreceptor mechanism. Hypotension that develops in such cases can be very prolonged, but usually recovers within 4 weeks with supportive treatment.

Among all the complications that happened following CBT resection, posterior circulation embolic strokes from the left CCA stump and prolonged persistent hypotension following left carotid body intervention in a normotensive individual are thought-provoking and worth mentioning. These events have not been reported in the literature to date. Both these complications can be well managed with medical treatment as described.

\section{SUPPLEMENTARY MATERIALS}

Supplementary materials related to this article can be found online at https://doi.org/10.5469/neuroint.2021.00248.

\section{Fund}

None.

\section{Ethics Statement}

Our institution IRB waives approval for case reports with less than 2 cases. Patient consent was obtained for the procedure and for publication of this report.

\section{Conflicts of Interest}

The authors have no conflicts to disclose.

\section{Author Contribution}

Concept and design: PKG. Analysis and interpretation: ST and PSK. Data collection: PKG and TSG. Writing the article: PKG, ST, and TSG. Critical revision of the article: PKG, ST, SP, AS, and
PSK. Final approval of the article: PKG, ST, TSG, SP, AS, and PSK. Overall responsibility: PKG and ST.

\section{ORCID}

Pawan K Garg: https://orcid.org/0000-0002-5805-1869

Sarbesh Tiwari: https://orcid.org/0000-0003-1661-9070

Tushar S Ghosh: https://orcid.org/0000-0001-5005-9236

Surendra Patel: https://orcid.org/0000-0003-1474-6738

Ankur Sharma: https://orcid.org/0000-0001-9339-6988

Pushpinder S Khera: https://orcid.org/0000-0002-9714-5462

\section{REFERENCES}

1. Texakalidis P, Charisis N, Giannopoulos S, Xenos D, Rangel-Castilla $L$, Tassiopoulos AK, et al. Role of preoperative embolization in carotid body tumor surgery: a systematic review and meta-analysis. World Neurosurg 2019;129:503-513.e2

2. Sylvester PT, Moran CJ, Derdeyn CP, Cross DT, Dacey RG, Zipfel GJ, et al. Endovascular management of internal carotid artery injuries secondary to endonasal surgery: case series and review of the literature. J Neurosurg 2016;125:1256-1276

3. Irvine CD. The significance of one occluded internal carotid artery. Eur J Vasc Endovasc Surg 1998;16:91-93

4. Cassidy L, Grace PA, Bouchier-Hayes DJ. The carotid stump syndrome. Eur J Vasc Surg 1992;6:368-370

5. Quill DS, Colgan MP, Sumner DS. Carotid stump syndrome: a colour-coded Doppler flow study. Eur J Vasc Surg 1989;3:79-83

6. Georgiadis D, Grosset DG, Lees KR. Transhemispheric passage of microemboli in patients with unilateral internal carotid artery occlusion. Stroke 1993;24:1664-1666

7. Fields WS, Lemak NA. Joint study of extracranial arterial occlusion. X. Internal carotid artery occlusion. JAMA 1976;235:27342738

8. Zhang XU, Shao S, Zheng X, Gao X, Zhang Y. Carotid stump syndrome: a case report. Exp Ther Med 2015;10:1161-1164

9. Dakhoul LT, Tawk R. Carotid stump syndrome: case report and endovascular treatment. J Investig Med High Impact Case Rep 2014:2:2324709614548796

10. Lakshminarayan R, Scott PM, Robinson GJ, Ettles DF. Carotid stump syndrome: pathophysiology and endovascular treatment options. Cardiovasc Intervent Radiol 2011;34 Suppl 2:S48-S52

11. Omoto S, Hasegawa Y, Sakai K, Matsuno H, Arai A, Terasawa Y, et al. Common carotid artery stump syndrome due to mobile thrombus detected by carotid duplex ultrasonography. J Stroke 
Cerebrovasc Dis 2016;25:e205-e207

12. Naylor AR, Bell PR, Bolia A. Endovascular treatment of carotid stump syndrome. J Vasc Surg 2003;38:593-595

13. Paivanas N, Bisognano J. Unilateral carotid body resection in resistant hypertension: an exciting first step toward a new therapy? JACC Basic Trans/ Sci 2016;1:325-327

14. Narkiewicz K, Ratcliffe LE, Hart EC, Briant LJ, Chrostowska M,
Wolf J, et al. Unilateral carotid body resection in resistant hypertension: a safety and feasibility trial. JACC Basic Trans/ Sci 2016;1:313-324

15. Fudim M, Groom KL, Laffer CL, Netterville JL, Robertson D, Elijovich F. Effects of carotid body tumor resection on the blood pressure of essential hypertensive patients. J Am Soc Hypertens 2015;9:435-442 